\title{
Confronting Scherk-Schwartz orbifold models with LHC data
}

\section{Dumitru Dan Smaranda*}

School of Physics and Astronomy - University of Glasgow, Glasgow, UK

E-mail: d.smaranda.1@research.gla.ac.uk

\section{David J Miller}

School of Physics and Astronomy - University of Glasgow, Glasgow, UK

E-mail: david.j.miller@glasgow.ac.uk

In the following proceedings we will outline our recent efforts aimed at analysing a class of models known as orbifold GUTs and their phenomenology in a variety of minimal and non-minimal settings. We examine the minimal $S U(5)$ models, rule them out, and proceed by extending them with an additional scalar field along with a gauge extension via $S U(5) \times U(1)$ models. We end up by commenting on the future improvements needed to more accurately handle exclusions along with tracing the $U(1)$ gauge extensions to more complete $6 D$ theories.

The 39th International Conference on High Energy Physics (ICHEP2018)

4-11 July, 2018

Seoul, Korea

${ }^{*}$ Speaker. 


\section{Introduction}

After the Higgs discovery, supersymmetry (SUSY) has had to face significant exclusions from the LHC data. Indeed the constrained Minimal Supersymmetric Standard Model, which gives all the supersymmetry breaking parameters a common value at a high scale, is almost completely ruled out [1]. However, typical supersymmetric models are complicated by having over 100 additional free parameters, so plenty of the parameter space remains to be explored. To this extent nonminimal supersymmetric models fit within these regions providing a continuing motivation for the LHC to search for them.

In this presentation we'll outline our efforts on how Scherk-Schwartz (SS) compactifications [2] affect a variety of extra dimensional GUT models with Kaluza Klein modes [3], and see if they agree with phenomenological constraints imposed by electroweak symmetry breaking and low energy experiments. We start of by exploring the basic model proposed in [4], and then move on to study scalar and $U(1)$ gauge extensions.

\section{Theory and Models}

Throughout this paper we'll be working on a $5 D$ compactified space $\mathscr{M}_{4} \times S^{1} / \mathbb{Z}_{2}$ with $\mathscr{N}=1$ SUSY. The SS action that we employ breaks the $5 D, \mathscr{N}=1$ supersymmetry to $4 D, \mathscr{N}=1$ on the brane at $y=0$, the Higgs flavour symmetry $S U(2)_{H} \rightarrow U(1)_{H}$ and the gauge symmetry $S U(5) \rightarrow$ $G_{S M}$ (note that we use the same gauge breaking for the $U(1)$ extension). The full form of $Z, T$ is the one used in [4].

Using these will in turn provide a soft SUSY breaking Lagrangain, which will depend on the fermionic matter placement (i.e. brane or bulk).

Since the basic model in [4] will fail to produce the right Higgs mass we'll move on and extend the Higgs sector via a scalar extension: $W=\lambda H_{u} H_{d} S+\frac{1}{3} \kappa S^{3}$, which will produce soft SUSY breaking masses from the SS action depending on the scalar placement (brane or bulk).

\section{Methodology and Constraints}

High scale parameters are introduced at the GUT scale and are run down to low energies using the FlexibleSUSY [v.2.0.1] [5] spectrum generator with two-loop Renormalisation Group Equations (RGEs), to produce electroweak symmetry breaking and a low energy spectrum. FlexibleSUSY relies on SARAH [v.4.12.2] [6] to generate the RGEs and the tadpole equations.

We check our model against LHC bounds and constraints from the ATLAS and CMS collaborations [7], which in our case comprise of: a Higgs mass between $123 \leq m_{H} \leq 127 \mathrm{GeV}$ (where we've assumed a $2 \mathrm{GeV}$ theoretical uncertainty arising from FlexibleSUSY)[8] ; a gluino mass larger than $m_{\tilde{g}} \geq 2 \mathrm{TeV}$ [9]; a neutralino mass larger than $m_{\tilde{\chi}_{1}^{0}} \geq 537$ for $\tan \beta \in[10,50]$ [10]; a stop mass larger than $m_{\tilde{t}} \geq 1 \mathrm{TeV}$ [11]; a chargino mass larger than $m_{\tilde{\chi}_{1}^{ \pm}} \geq 460 \mathrm{GeV}$ [12]; an extra $Z^{\prime}$ gauge boson with a mass larger than $m_{Z^{\prime}} \geq 2.4 \mathrm{TeV}$ [13] for the $U(1)$ extensions.

To check the dark matter relic density we use MicrOmegas [14]. The dark matter relic density bound is in accordance with the latest Planck data [15] : $\Omega_{c} h^{2}=0.1157 \pm 0.0023$, where we consider a $10 \%$ uncertainty from the mass difference from MicrOmegas and FlexibleSUSY, and accept all points with a dark matter relic density smaller than $\Omega_{c} h^{2}=0.1275$. 


\section{Results and Conclusions}

Throughout our scans we found that the basic $S U(5)$ model proposed in [4] cannot produce the appropriate Higgs mass with brane or bulk matter. We then moved on to trying a scalar extension of the $S U(5)$ model which yielded in a better result concerning the Higgs, but failed in the end to meet the SS constraint. This will be further explored in future work to try and more accurately quantify the SS uncertainty resulting from threshold corrections. Furthermore to try and bypass the SS constraint we looked at a scalar extension with a trivialised $S U(2)_{H}$ symmetry. In this case we got the right Higgs mass but the model was eliminated due to LHC cuts and dark matter relic density constraints.

Finally the additional scalar within the $U(1)_{N}$ extension framework resulted in a similar scenario where the points that obeyed the SS constraint did not produce an appropriate Higgs mass (see Figure 1). This has in turn prompted future work in which we will treat the spectrum in Figure 1 as a remnant of a $6 D, E_{6}$ theory (e.g. as in [16]) which would arise from a more complicated accidental flavour symmetry induced by the different $27, \overline{27}$ representations delivering "a different SS constraint".

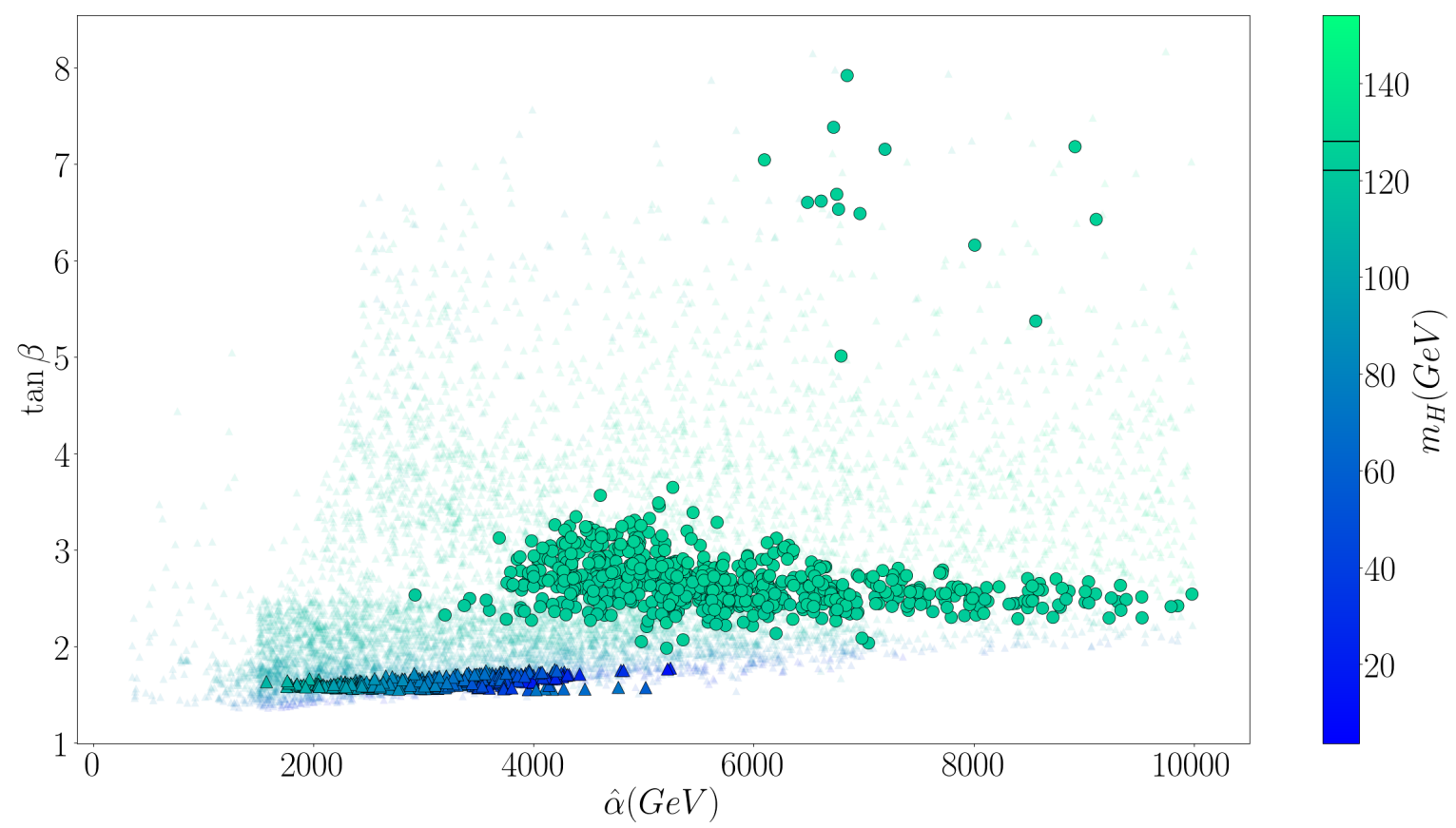

Figure 1: $S U(5) \times U(1)_{N}$ plus bulk Scalar $\mathscr{S}$ with brane matter $T, F$. The points marked by triangles represent points originating from SS breaking, and the points marked by circles represent points with the right Higgs mass. The transparent points produce EWSB but do not pass LHC constraints.

To summarise, the basic model cannot produce the right Higgs mass, the naive scalar extensions don't quite work and we are looking on quantifying threshold effects along with exploring more complicated theories that can accommodate SS breaking.

\section{References}

[1] ATLAS collaboration, G. Aad et al., Summary of the searches for squarks and gluinos using $\sqrt{s}=8$ 
TeV pp collisions with the ATLAS experiment at the LHC, JHEP 10 (2015) 054 [1507. 05525].

[2] J. Scherk and J. H. Schwarz, Spontaneous breaking of supersymmetry through dimensional reduction, Physics Letters B 82 (1979) 60 . J. Scherk and J. H. Schwarz, How to get masses from extra dimensions, Nuclear Physics B 153 (1979) 61 .

[3] T. Kaluza, Zum Unitätsproblem der Physik, Sitzungsber. Preuss. Akad. Wiss. Berlin (Math. Phys.) 1921 (1921) 966 [1803.08616]. O. Klein, Quantum Theory and Five-Dimensional Theory of Relativity. (In German and English), Z. Phys. 37 (1926) 895.

[4] R. Barbieri, L. J. Hall and Y. Nomura, Softly broken supersymmetric desert from orbifold compactification, Phys. Rev. D66 (2002) 045025 [hep-ph/ 0106190 ]. R. Barbieri, L. J. Hall and Y. Nomura, Models of Scherk-Schwarz symmetry breaking in 5-D: Classification and calculability, Nucl. Phys. B624 (2002) 63 [hep-th/0107004].

[5] P. Athron, J.-h. Park, D. Stöckinger and A. Voigt, FlexibleSUSY-A spectrum generator generator for supersymmetric models, Comput. Phys. Commun. 190 (2015) 139 [1 406 . 2319].

[6] F. Staub, SARAH, 0806.0538.

[7] ATLAS-SUSY, Atlas susy summary plots, . ATLAS-Exotics, Atlas exotics summary plots, . CMS-SUSY, Cms susy summary plots, .

[8] ATLAS collaboration, G. Aad et al., Observation of a new particle in the search for the Standard Model Higgs boson with the ATLAS detector at the LHC, Phys. Lett. B716 (2012) 1 [1207. 7214 ]. CMS collaboration, S. Chatrchyan et al., Observation of a new boson at a mass of $125 \mathrm{GeV}$ with the CMS experiment at the LHC, Phys. Lett. B716 (2012) 30 [1207 . 7235].

[9] ATLAS collaboration, M. Aaboud et al., Search for squarks and gluinos in final states with jets and missing transverse momentum using $36 \mathrm{fb}^{-1}$ of $\sqrt{\mathrm{s}}=13 \mathrm{TeV}$ pp collision data with the ATLAS detector, Phys. Rev. D97 (2018) 112001 [1712. 02332]. CMS collaboration, A. M. Sirunyan et al., Search for supersymmetry in proton-proton collisions at $13 \mathrm{TeV}$ using identified top quarks, Phys. Rev. D97 (2018) 012007 [1710.11188].

[10] ATLAS collaboration, G. Aad et al., Searches for heavy long-lived charged particles with the ATLAS detector in proton-proton collisions at $\sqrt{s}=8 \mathrm{TeV}$, JHEP 01 (2015) 068 [1411.6795].

[11] ATLAS collaboration, M. Aaboud et al., Search for top-squark pair production in final states with one lepton, jets, and missing transverse momentum using $36 \mathrm{fb}^{-1}$ of $\sqrt{\mathrm{s}}=13 \mathrm{TeV}$ pp collision data with the ATLAS detector, JHEP 06 (2018) 108 [1711.11520].

[12] ATLAS collaboration, M. Aaboud et al., Search for long-lived charginos based on a disappearing-track signature in pp collisions at $\sqrt{s}=13 \mathrm{TeV}$ with the ATLAS detector, JHEP 06 (2018) 022 [1712.02118].

[13] ATLAS collaboration, M. Aaboud et al., Search for additional heavy neutral Higgs and gauge bosons in the ditau final state produced in $36 \mathrm{fb}^{-1}$ of pp collisions at $\sqrt{\mathrm{s}}=13 \mathrm{TeV}$ with the ATLAS detector., JHEP 01 (2018) 055 [1709.07242].

[14] D. Barducci, G. Belanger, J. Bernon, F. Boudjema, J. Da Silva, S. Kraml et al., Collider limits on new physics within micrOMEGAs4.3, 1606.03834.

[15] G. Hinshaw, D. Larson, E. Komatsu, D. N. Spergel, C. L. Bennett, J. Dunkley et al., Nine-year wilkinson microwave anisotropy probe (wmap) observations: Cosmological parameter results, 1212.5226.

[16] R. Nevzorov, $E_{6}$ inspired supersymmetric models with exact custodial symmetry, Phys. Rev. D87 (2013) 015029 [1205.5967]. 